\title{
Critical behavior of the magnetic susceptibility of the uniaxial ferromagnet LiHoF4
}

\author{
Beauvillain, P.; Renard, J. P.; Laursen, lb; Walker, P. J.
}

Published in:

Physical Review B

Link to article, DOI:

10.1103/PhysRevB.18.3360

Publication date:

1978

Document Version

Publisher's PDF, also known as Version of record

Link back to DTU Orbit

Citation (APA):

Beauvillain, P., Renard, J. P., Laursen, I., \& Walker, P. J. (1978). Critical behavior of the magnetic susceptibility of the uniaxial ferromagnet LiHoF4. Physical Review B, 18(7), 3360-3368.

https://doi.org/10.1103/PhysRevB.18.3360

\section{General rights}

Copyright and moral rights for the publications made accessible in the public portal are retained by the authors and/or other copyright owners and it is a condition of accessing publications that users recognise and abide by the legal requirements associated with these rights.

- Users may download and print one copy of any publication from the public portal for the purpose of private study or research.

- You may not further distribute the material or use it for any profit-making activity or commercial gain

- You may freely distribute the URL identifying the publication in the public portal

If you believe that this document breaches copyright please contact us providing details, and we will remove access to the work immediately and investigate your claim 


\title{
Critical behavior of the magnetic susceptibility of the uniaxial ferromagnet $\mathrm{LiHoF}_{4}$
}

\author{
P. Beauvillain and J.-P. Renard \\ Institut d'Electronique Fondamentale, Laboratoire associe au Centre National de la Recherche Scientifique \\ Bâtiment 220, Université Paris-Sud, 91405 Orsay Cédex, France \\ I. Laursen \\ Department of Electrophysics, Building 322, The Technical University of Denmark, DK-2800, Lyngby, Denmark \\ P. J. Walker \\ Clarendon Laboratory, Parks Road, Oxford, United Kingdom
}

(Received 21 February 1978).

\begin{abstract}
The magnetic susceptibility of two $\mathrm{LiHoF}_{4}$ single crystals has been measured in the range $1.2-4.2 \mathrm{~K}$. Ferromagnetic order occurs at $T_{c}=1.527 \mathrm{~K}$. Above $2.5 \mathrm{~K}$, the susceptibilities parallel and perpendicular to the fourfold $c$ axis are well interpreted by the molecular-field approximation, taking into account the ground state and the first excited state of $\mathrm{Ho}^{3+}$ in the crystal field of $S_{4}$ symmetry. The experimental results are consistent with $g_{\|}=13.95$ and $g_{\perp}=0$ for the ground state. The dipolar contribution to the magnetic interaction is about three times larger than the exchange one. Near $T_{c}$, the parallel susceptibility is well described by the classical law with logarithmic corrections theoretically predicted by Larkin and Khmel'mitskii for the uniaxial dipolar ferromagnet or by a power law with a critical-exponent value $\gamma=1.05$ rather close to 1 . The upper limit of the critical region is $\left(T_{\max }-T_{c}\right) / T_{c}=1.1 \times 10^{-2}$.
\end{abstract}

\section{INTRODUCTION}

The compounds $\mathrm{Li} R_{x} \mathrm{Y}_{1-x} \mathrm{~F}_{4}$ where $R$ is a rare earth, crystallized in the tetragonal scheelite structure $I 4_{1 / a}{ }^{1}$ They are efficient laser materials ${ }^{2}$ and can be used for frequency conversion in the infrared-visible region. $^{3}$ Due to their practical interest, many experimental studies have been done on these materials: chiefly, absorption and fluorescence ${ }^{4}$ and electron paramagnetic resonance (EPR).$^{5-7}$

Another exciting characteristic of these compounds, especially those with $100 \%$ rare earth, is their low-temperature magnetic behavior. Indeed, one can expect that the rare-earth atoms which are fairly ionic are predominantly coupled by dipoledipole interaction. Moreover, the high-point symmetry through the rare earth and the relative simplicity of the crystal structure allow theoretical calculations. Recently, Misra and Felsteiner ${ }^{8}$ have theoretically studied the magnetically ordered state of the $\mathrm{Li} \mathrm{F}_{4}$ by a generalization of the Luttinger-Tisza method, considering only dipole-dipole interactions between rare-earth ions. They predicted ferromagnetism for $g_{\|}>g_{\perp}$ and antiferromagnetism for $g_{\|}<g_{\perp}$ where $g_{\|}$and $g_{1}$ are, respectively, the Landé tensor component parallel and perpendicular to the $c$ axis. At this time, the magnetic phase transition has been observed only for $R=\mathrm{Tb}, \mathrm{Ho}$, and $\mathrm{Er}$. Both $\mathrm{LiTbF}_{4}$ $\left(T_{c}=2.874 \mathrm{~K}\right)^{9}$ and $\mathrm{LiHoF}_{4}\left(T_{c}=1.53 \mathrm{~K}\right)^{10,11}$ are ferromagnets with the easy axis along $c$ and $\mathrm{LiErF}_{4}$ was found to order antiferromagnetically ${ }^{12}\left(T_{N}=0.381 \mathrm{~K}\right)$ with the spins lying in the $a a$ plane. This is consistent with the theoretical predictions since for $\mathrm{LiErF}_{4} g_{\perp} \gg g_{\|}$, while for $\mathrm{LiTbF}_{4}$ and $\mathrm{LiHoF}_{4} g_{\perp}$ is close to zero and $g_{\|}$is rather large, respectively, 17.85 and 14.1. ${ }^{6}$ Both $\mathrm{LiTbF}_{4}$ and $\mathrm{LiHoF}_{4}$ appear as nearly ideal uniaxial ferromagnets.

Critical phenomena in such uniaxial systems with dipolar interactions are extremely interesting. Indeed, in this special case the marginal dimensionality $d^{*}$ above which the critical behavior is classical, i.e., given by the Landau theory, is $d^{*}=3$ instead of $d^{*}=4$ for systems with short-range interactions. ${ }^{13}$ At the marginal dimensionality, Larkin and

Khmel'nitskii ${ }^{14}$ (LK) predicted logarithmic correction terms to the Landau-like critical behavior. This was further supported by Aharony ${ }^{15}$ and Brézin and Zinn-Justin ${ }^{16}$ for uniaxial dipolar ferromagnets.

In particular, the susceptibility should diverge as $t^{-1}|\ln t|^{1 / 3}$ rather than as the Landau form of $t^{-1}, t$ being the reduced temperature $\left|T-T_{c}\right| / T_{c}$. One difficulty in observing logarithmic corrections to the mean-field power laws arises from the fact that these corrections are only valid in the limit of $T \rightarrow T_{c}$ whereas experiments are always carried out at a finite distance from $T_{c}$. Aharony and Halperin ${ }^{17}$ have suggested from their renormalization group (RG) analysis that the form $\ln \left(t / t_{0}\right)$ is used to analyze experimental data with $t_{0}$ being an adjustable parameter. Detailed comparisons between RG theory and experi- 
ments on $\mathrm{LiTbF}_{4}$ are now available. In the specific heat the logarithmic divergence is the leading singularity and high-precision experiments of Ahlers et al. ${ }^{18}$ were indeed consistent with a $\left|\ln \left(t / t_{0}\right)\right|^{1 / 3}$ behavior and with an amplitude ratio of $\frac{1}{4}$ for the specific heat above and below $T_{c}$ as predicted. Aharony and Halperin ${ }^{17}$ pointed out that the RG equations implied an exact relationship between the spin correlation range and the specific-heat data. The neutron scattering data by Als-Nielsen ${ }^{19}$ confirmed this relation accurately. In addition he found that the amplitude ratio of the susceptibility above and below $T_{c}$ had the mean-field and RG value of 2 . Finally, very accurate spontaneous magnetization data were obtained by Griffin et al. ${ }^{20}$ using Faraday rotation, and although the leading singularity here is a power law with exponent $\beta$, they concluded that $\beta=\frac{1}{2}$ with logarithmic corrections did fit the data better than the best-fit power law with $\beta=0.38$.

The compounds $\mathrm{Li} \mathrm{F}_{4}$ are fair but not ideal model systems of the uniaxial dipolar coupled ferromagnet. With $R=\mathrm{Tb}$ the ground state is actually not an Ising doublet, but is split by a small amount due to the crystal field. Higher excited states are present albeit at energies considerably above $k_{B} T_{c}$, and finally exchange interactions are present in addition to the dipolar interaction. All these effects are different in $\mathrm{LiHoF}_{4}$, and we have therefore initiated a study of the critical behavior of this substance to supplement the information available from $\mathrm{LiTbF}_{4}$.

We report here measurements of parallel and perpendicular susceptibility of $\mathrm{LiHoF}_{4}$ in order to define the Landé tensor components $g_{\|}$and $g_{\perp}$ and the exchange and dipolar contributions to magnetic interaction between rare-earth ions. Since these measurements confirmed that $\mathrm{LiHoF}_{4}$ is a rather good uniaxial dipolar ferromagnet, we performed very accurate parallel susceptibility measurements in the critical region and tried to discriminate between the theoretically predicted classical law with logarithmic corrections $t^{-1}\left|\log _{10} t\right|^{1 / 3}$ and the usual power law $t^{-\gamma}$.

\section{EXPERIMENTAL TECHNIQUES}

\section{A. Crystal growth and sample preparation}

The measurements were performed on two different $\mathrm{LiHoF}_{4}$ samples. Sample 1 was grown at the Lyngby Technical University of Denmark by the method previously described by Laursen and Holmes. ${ }^{7}$ It was ground into an ellipsoid of $7.26 \times 1.92 \times 1.24 \mathrm{~mm}^{3}$ with its long axis parallel to $c$, weighing $53.2 \pm 0.2 \mathrm{mg}$.

Sample 2 was grown by Walker at Oxford Clarendon Laboratory using also the Stockbarger method. A monocrystalline part of optical quality was selected and ground into a sphere of $4.836 \pm 0.002-\mathrm{mm}$ diam and $339.8 \pm 0.2-\mathrm{mg}$ weight. In both cases, the starting materials were of purity $>99.9 \%$ and the crystal quality was controlled by $\mathrm{x}$-ray diffraction.

\section{B. Susceptibility measurements and thermometry}

Magnetic susceptibility was measured by means of an ac mutual inductance bridge ${ }^{21}$ operating at $70 \mathrm{~Hz}$. The susceptibility probe was calibrated against chromium-potassium alum with a precision of about $1 \%$. The amplitude of the ac measuring field was kept as low as 2 Oe in order to avoid nonlinear effects, especially close to $T_{c}$. The bridge balance was achieved by sample extraction. The sample and the probe were directly immersed in a pumped ${ }^{4} \mathrm{He}$ bath. Above $2.17 \mathrm{~K}$, helium pressure was stabilized by a Cartesian manostat. Below $2.17 \mathrm{~K}$, an electronic stabilization of the ${ }^{4} \mathrm{He}$ bath was achieved by a commerical temperature controller ${ }^{22}$ (ac resistance bridge ATNE). The temperature sensor was a $47-\Omega, \frac{1}{8}-W$ Allen Bradley carbon resistor. Temperature stability was better than $0.1 \mathrm{mK}$ in this range. Temperature was obtained from a germanium resistor ${ }^{23}$ thermally anchored to the sample and its Teflon holder by a bundle of 200 thin copper wires. It was measured with an ATNE ac resistance bridge and calibrated against the ${ }^{4} \mathrm{He}$ vapor pressure using $T_{58}$ scale. The ${ }^{4} \mathrm{He}$ pressure was determined from an oil manometer and a commercial pressure gauge. ${ }^{24}$ Above $2.17 \mathrm{~K}$, ${ }^{4} \mathrm{He}$ is not superfluid and its pressure corresponds to the surface temperature which apprecially differs from that inside the bath. In this range the germanium resistor was calibrated against the magnetic susceptibility of $\mathrm{CrK}$ alum. The reproducibility and the precision of the thermometer are discussed in detail in Sec. III.

\section{Sample orientation}

Sample 1 (ellipsoid) was oriented by the conventional Laue- $x$-ray technique and set in a long cylindrical holder inside the susceptibility probe. The spherical sample 2 was set in a Teflon conical holder and its orientation was achieved in situ by applying a dc magnetic field. Since $g_{\|} \gg g_{1}$, fields of about $200 \mathrm{Oe}$ were large enough to orientate the $c$ axis along the field with a precision better than $1^{\circ}$ at $4.2 \mathrm{~K}$. Vertical and horizontal dc fields were, respectively, produced by a solenoid coaxial to the susceptibility probe and a pair of Helmoltz coils.

\section{EXPERIMENTAL RESULTS AND DISCUSSION}

\section{A. Parallel and perpendicular susceptibilities} in the range $1.2-4.2 \mathrm{~K}$

The measured susceptibilities per gram $\chi_{11}^{m}$, and $\chi_{1}^{m}$ of sample 2 , in the temperature range $1.2-4.2 \mathrm{~K}$ are 
given in Fig. 1. $\chi_{\perp}^{m}$ is much smaller than $\chi_{\|}^{m}$. This reflects the strong anisotropy of the $g$ tensor in the ground state. $\chi_{11}^{m}$ reaches a plateau below

$T_{c}=1.526 \pm 0.004 \mathrm{~K}$ at a maximum value of $(4.195 \pm 0.040) \times 10^{-2}$ emu cgs which corresponds to $1 / N \rho=4.175 \times 10^{-2}$ emu cgs, where $N=\frac{4}{3} \pi$ is the sphere demagnetizing factor and $\rho=5.72$ the crystal density. This variation of $\chi_{\| 1}^{m}$ versus temperature is consistent with a transition at $T_{c}$ to a ferromagnetic state with the $\mathrm{Ho}^{3+}$ spins along the $c$ crystal axis, in agreement with previous measurements by Cooke et al. ${ }^{11}$ and Hansen et al. ${ }^{10}$

To explain the experimental data well above $T_{c}$ $(2.5-4.2 \mathrm{~K})$, we used the $\mathrm{Ho}^{3+}$ energy scheme in $\mathrm{LiHoF}_{4}$ proposed by Hansen et al. ${ }^{10}$ and Margariño and Tuchendler. ${ }^{25}$ In a crystal field of $S_{4}$ symmetry, the $(2 J+1)$-fold degenerate ground term ${ }^{5} I_{8}$ is split into a fundamental doublet $\Gamma_{3,4}$ and a first excited $\Gamma_{2}$ singlet of energy $E_{1} / k_{B}=10.4 \pm 0.4 \mathrm{~K}$. The other excited states are well above and they only give the temperature-independent Van Vleck contributions to low-temperature susceptibility. A straightforward calculation leads to the following expression for the parallel susceptibility $\chi_{\text {is }}^{m}$ of the isolated $\mathrm{Ho}^{3+}$ ion (neglecting the interactions):

$$
\chi_{\mathrm{is}}^{m}=\frac{n \mu_{B}^{2}}{4 k} \frac{\left(g_{\|}^{0}\right)^{2} T^{-1}+a_{\|}^{0}+a_{\|}^{1} e^{-E_{1} / k T}}{1+0.5 e^{-E_{1} / k T}},
$$

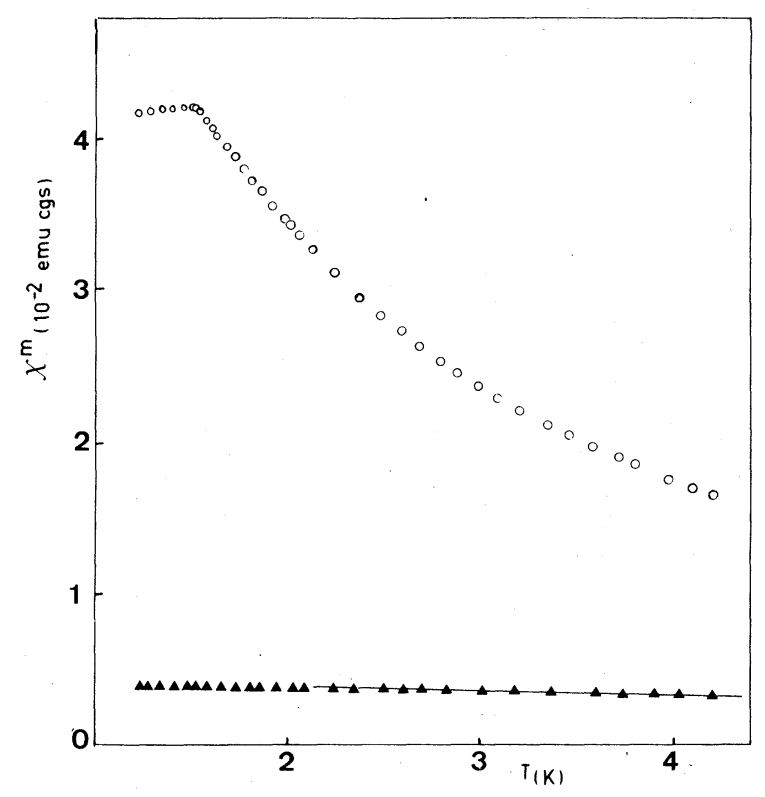

FIG. 1. Experimental parallel susceptibility per gram $\chi_{\|}^{m}$ (open circles) and perpendicular susceptibility per gram $\chi_{\perp}^{m}$ (black triangles) vs temperature for the spherical sample. The solid line represents the approximate theoretical law, for $e^{-E_{1} / k T}<<1: \chi_{\perp}^{m}=\left(n \mu_{B}^{2} / 4 k\right)\left(B+C e^{-E_{1} / k T}\right)$, with $B=9.98, C=11.3$, and $E_{1} / k=1,0.4 \mathrm{~K}$. where $n$ is the number of Ho atoms per gram, $\mu_{B}$ the Bohr magneton, $k$ the Boltzman constant, $g_{\|}^{0}$ the Landé tensor component along $c$ for the ground state, and $a_{\|}^{0}, a_{\|}^{1}$ the respective Van Vleck contributions of the ground state and of the first excited state. When we take into account the interaction between $\mathrm{Ho}^{3+}$ ions in a simple mean-field approximation, we obtain for $\chi_{\|}^{m}$

$$
\chi_{\|}^{m}=\chi_{\mathrm{is}}^{m} /\left(1-\alpha_{\|} \chi_{\mathrm{is}}^{m}\right),
$$

where the parameter $\alpha_{\|}$contains the dipolar and exchange contributions to the interaction (see Fig. 2).

The best fit of relations (1) and (2) to the experimental data between $2.5 \mathrm{~K}$ and $4.2 \mathrm{~K}$ is achieved for $g_{\|}^{0}=13.95 \pm 0.15, a_{\|}^{0}=0.25 \pm 0.1, a_{\|}^{1}=3.3 \pm 1.5$, and $\alpha_{\|}=-1.6 \pm 0.4$ (emu cgs) ${ }^{-1}$. The $g_{\|}^{0}$ value is in good agreement with the EPR one ${ }^{6} g_{11}^{0}=14.1 \pm 0.2$ but it differs significantly from that of $\mathrm{Ho}^{3+}$ diluted in $\mathrm{LiYF}_{4}: g_{\|} 0=13.3 \pm 0.1$. It also slightly differs from the value of $13.6 \pm 0.2$ determined from previous susceptibility measurements. ${ }^{11}$ However, these measure-

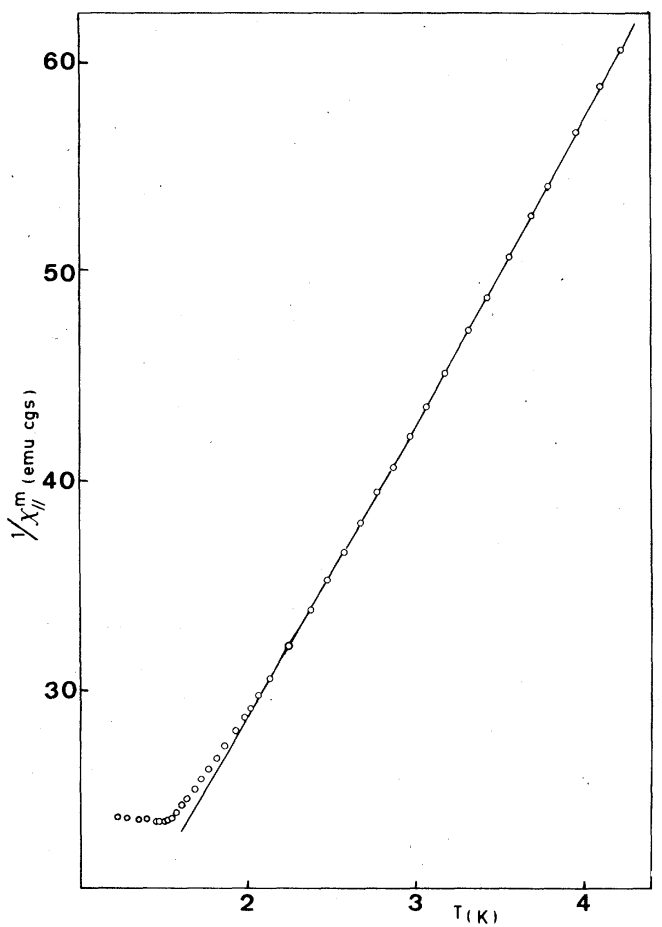

FIG. 2. Reciprocal experimental parallel susceptibility per gram $1 / X_{\|}^{m}$ vs temperature for a spherical sample. The solid line represents the theoretical curve $1 / \chi_{\|}^{m}=1 / \chi_{\text {is }}^{m}-\alpha_{\|}$ with

$$
\chi_{\mathrm{is}}^{m}=\frac{n \mu_{B}^{2}}{4 k}\left[\frac{\left(g_{\|}^{0}\right)^{2} T^{-1}+a_{\|}^{0}+a_{\| \ell}^{1} e^{-E_{1} / k T}}{1+0.5 e^{-E_{1} / k T}}\right]
$$

with $\alpha_{\|}=-1.6, g_{\|}^{0}=13.95, a_{\|}^{0}=0.25, a_{\|}^{1}=3.3$, and $E_{1} / k=10.4 \mathrm{~K}$ 
ments were interpreted by the simple Curie-Weiss law: $\chi_{\|}^{m}=C_{m} / T-\Theta$. Fitting our experimental data with this Curie-Weiss law in the range $2.5-4.0 \mathrm{~K}$ leads to $g_{\|}=13.55 \pm 0.1, C_{m}=(6.95 \pm 0.1) \times 10^{-2}$ emu cgs and $\Theta=+0.02 \pm 0.04 \mathrm{~K}$ in good agreement with Ref. 11. Our value of $a_{\|}^{1}$ is rather imprecise and will be improved by further $\chi_{\|}$measurements above $4.2 \mathrm{~K}$.

The $\alpha_{\|}$value can be easily related to dipolar and exchange interactions. The local field $\overrightarrow{\mathrm{H}}_{\mathrm{loc}}$ at a rareearth-ion site is given by

$$
\overrightarrow{\mathrm{H}}_{\mathrm{loc}}=\left[1+\left(\frac{4}{3} \pi \rho-\vec{\delta}+\vec{\epsilon}-\overrightarrow{\mathrm{N}} \rho\right) \cdot \vec{\chi}\right] \cdot \overrightarrow{\mathrm{H}},
$$

where $\overrightarrow{\mathrm{H}}$ is the external field, $\vec{\chi}$ the susceptibility tensor, and $\vec{\delta}, \vec{\epsilon}$, and $\vec{N}$ are, respectively, the dipolar, exchange, and demagnetizing field tensor. $\vec{\delta}$ is expressed as follows:

$$
\vec{\delta}=n^{-1} \sum_{i} \frac{\overrightarrow{1} r_{i}^{2}-3 \vec{r}_{i} \overrightarrow{\mathrm{r}}_{i}}{r_{i}^{5}},
$$

where $\vec{r}_{i}$ is the vector to the $i$ th rare-earth neighbor.

Due to the $S_{4}$ symmetry, all tensors are diagonal in a coordinate system which contains the fourfold axis c. For a spherical sample $\vec{N}=\frac{4}{3} \pi \overrightarrow{1}$ and $\alpha_{\|}$is simply given by

$$
\alpha_{\|}=\epsilon_{\|}-\delta_{\|},
$$

where $\epsilon_{\|}$and $\delta_{\|}$denote the tensor components along c.

$\delta_{\|}$has been calculated from (4) by summing over the $\mathrm{Ho}^{3+}$ sites inside a sphere of $400-\AA$ diam. Taking into account the obtained value of $\delta_{\|}=-9.5$ and the experimental $\alpha_{\|}$, we determined $\epsilon_{\|}=-11.1 \pm 0.4$. This exchange contribution is much smaller than the total dipolar one: $\frac{4}{3} \pi \rho-\delta_{\|}=33.46$. The corresponding exchange energy in the ground state $z J / 4 k=n \epsilon_{\|}\left(g_{\|}^{0} \mu_{B}\right)^{2} / 4 k=-0.820 \pm 0.03 \mathrm{~K}$ may be compared to that of $\mathrm{LiErF}_{4}: z J / 4 k=-0.620 \mathrm{~K}$. We can also describe the mass perpendicular susceptibility $\chi_{\perp}^{m}$ using formulas (1) and (2) with the character $\perp$ instead of II. Since $g_{1}=0$ and $\exp \left(-E_{1} / k T\right)<<1, \chi_{\perp}^{m}$ takes the approximate form

$$
\chi_{\perp}^{m}=\frac{n \mu_{B}^{2}}{4 k}\left(B+C e^{-E_{1} / k T}\right)
$$

with

$B=a_{\perp}^{0}\left(1+\alpha_{1} A a_{\perp}^{0}\right)$ and $C=a_{\perp}^{1}+a_{\perp}^{0}\left(\alpha_{1} A a^{1}-0.5\right)$

with $A=n \mu_{B}^{2} / 4 k$.

The best fit in the range $2.8-4.2 \mathrm{~K}$ is obtained for $B=9.98 \pm 0.05$ and $C=11.3 \pm 0.7$. The present temperature range is too restricted to obtain independently $a_{\perp}^{0}, a_{\perp}^{1}$, and $\alpha_{\perp}$.

\section{B. Critical behavior of the parallel susceptibility}

We have measured accurately the parallel susceptibility of $\mathrm{LiHoF}_{4}$ in the range $1.5-1.8 \mathrm{~K}$. Two and four successive experimental runs were done, respectively, on samples 1 and 2 . Temperature of the sample and thermometer was kept below $77 \mathrm{~K}$ between runs in order to avoid calibration drift and nonreproducibleness of the thermometer. Each run contained about 60 points. Helium vapor pressure was carefully measured at about 20 points for calibrating the germanium resistor thermometer. In the considered temperature range, the experimental values $T(R)$ are accurately fitted by the empirical Clement and Quinnel relation (Fig. 3):

$$
T^{-1}=a \log _{10} R+b\left(\log _{10} R\right)^{-1}+c .
$$

The parameters $a, b, c$ were obtained by the leastsquares method. For each selected calibration point, the difference between temperatures obtained from the $T_{58}$ scale and from relation (6) is less than 0.3 $\mathrm{mK}$.

The measured parallel susceptibility $\chi_{11}^{m}$ of sample 2 in the temperature range $1.52-1.65 \mathrm{~K}$ is shown on Fig. 4. Owing to the resolution of the ac mutual inductance bridge of $4 \times 10^{-7}$ emu cgs, the data of the four different experimental runs are very well consistent with each other.

We have compared the experimental $\chi_{\| 1}^{m}$ data for both samples 1 and 2 to the classical law with logarithmic corrections theoretically predicted by $L^{14}$ :

$$
\chi_{c}=A t^{-1}\left|\log _{10} t\right|^{1 / 3}
$$

and to the power law

$$
\chi_{c}=A^{\prime} t^{-\gamma},
$$

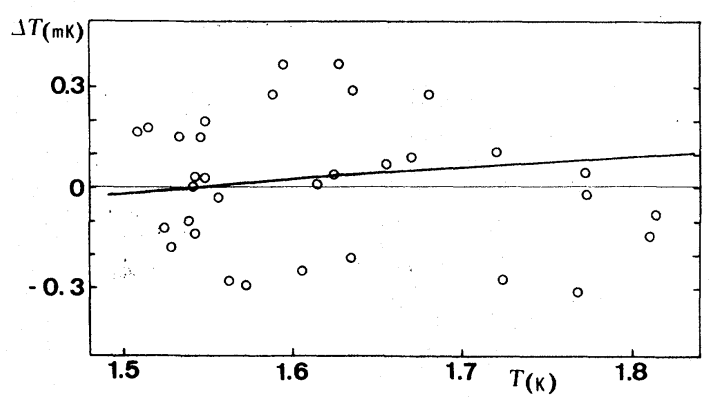

FIG. 3. Plot of $\Delta T$, the difference between temperatures obtained from the $T_{58}$ scale and from the empirical Clement and Quinnel relation (1) $T^{-1}=a \log _{10} R+b\left(\log _{10} R\right)^{-1}+c$ with $a=0.749801, b=7.578944$, and $c=-4.439235$ vs temperature in the range $1.5-1.8 \mathrm{~K}$. The solid line represents the difference between temperatures obtained with relation (1) for two different sets of parameters: the $a, b$, and $c$ values reported above and $a^{\prime}=0.750987, b^{\prime}=7.599984$, $c^{\prime}=-4.449258$ corresponding to least-squares fit for, respectively, four and three different runs. 


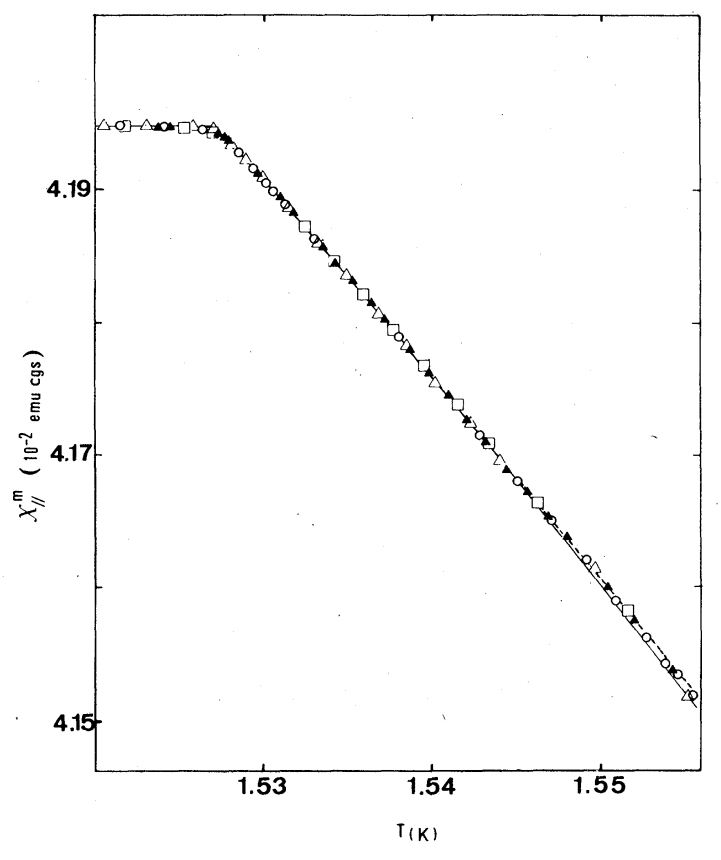

FIG. 4. Experimental parallel susceptibility per gram vs temperature in the range $1.52-1.56 \mathrm{~K}$ for four different runs on the spherical sample. Full line represents the classical law with logarithmic corrects and dashed line the power law with parameter values of Table $\mathrm{I}$. where $t$ is the reduced temperature $t=\left(T-T_{c}\right) / T_{c}$ and $\chi_{c}$ the susceptibility corrected from the demagnetizing field effect. $\chi_{\max }$ being the maximum value of $\chi_{\| 1}^{m}$, we define $\chi_{\mathrm{th}}(t)$ by

$$
\left[\chi_{\mathrm{th}} t\right]^{-1}=\chi_{c}^{-1}(t)+\chi_{\max }^{-1} .
$$

In the case of the theoretical LK law (7), we have to adjust three parameters: $\chi_{\max }, T_{c}$, and $A$ for the best fit of $\chi_{\text {th }}$ to the experimental data. We also introduce two additional parameters $T_{\min }$ and $T_{\max }$ which are the limits of the temperature range of the fit. One of these parameters $\chi_{\max }$ is relatively well defined from the susceptibility measurements just below $T_{c}$. Thus for a given value of $\chi_{\max }$, and for a series of $T_{c}$ values, we determine the $A$ values which give the best fit to the experimental data in the range $T_{\min }<T<T_{\max }$.

In Fig, 5, we have plotted the average difference

$$
\Delta=\frac{1}{N} \sum_{i}\left|\chi_{\mathrm{th}}\left(t_{i}\right)-\chi_{\|}^{m}\left(t_{i}\right)\right|,
$$

where $N$ is the number of experimental points between $T_{\min }$ and $T_{\max }$ versus $A$. For a given $T_{c}, A$ is determined as the value which corresponds to the minimum of $\Delta(A)$. The best $T_{c}$ value corresponds to the $\Delta(A)$ curve which has the lower minimum. For various intervals $T_{\min }-T_{\max }$, the best $T_{c}$ and $A$ values are obtained by this procedure. We observed that these $T_{c}$ and $A$ values do not depend on the interval $T_{\min }-T_{\max }$ provided that $T_{\min }$ is outside the

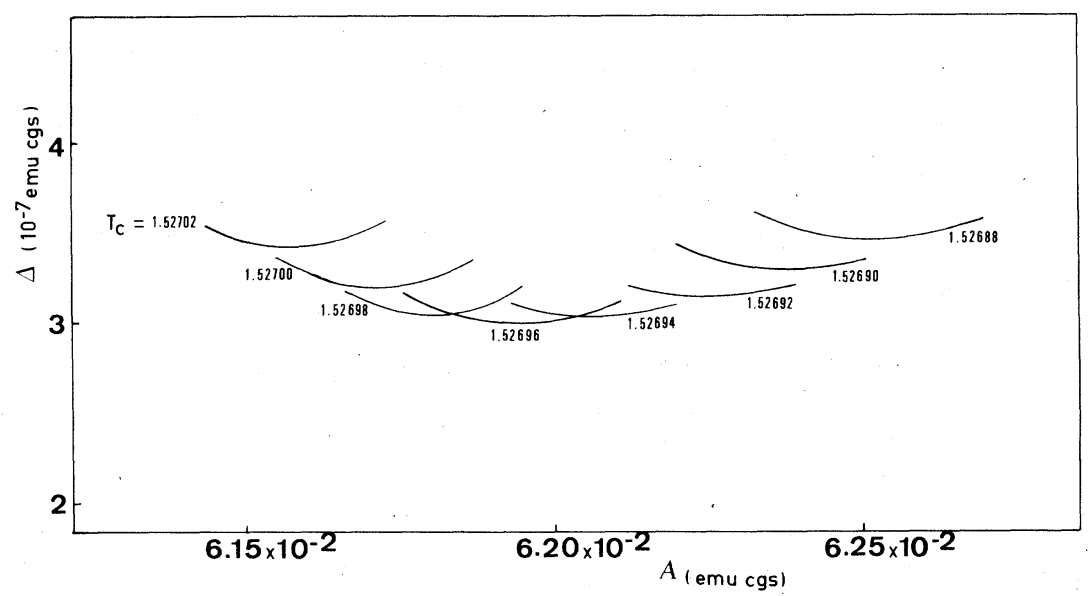

FIG. 5. Average difference $\Delta$ vs critical amplitude $A$ for different values of $T_{c}$ and for a given value of $\chi_{\max }=4.1952 \times 10^{-2}$ emucgs and $T_{\max }=1.544 \mathrm{~K}, T_{\min }=1.5273 \mathrm{~K}$.

$$
\Delta=(1 / N) \Sigma_{i}\left|\chi_{\mathrm{th}}\left(t_{i}\right)-\chi_{\|}^{m}\left(t_{i}\right)\right| \text {, }
$$

where $N$ is the number of experimental points in the range $T_{\min }<T<T_{\max } . \quad \chi_{\|}^{m}$ is the parallel susceptibility per gram of the spherical sample and $\chi_{\text {th }}$ is the theoretical susceptibility for a critical behavior with logarithmic corrections:

$$
1 / \chi_{\mathrm{th}}(t)=1 / A t^{-1}\left|\log _{10} t\right|^{1 / 3}+1 / \chi_{\max }
$$

The best fit is obtained for $T_{c}=1.52696$ and $A=6.195 \times 10^{-2}$. 
TABLE I. Critical temperature and amplitude for the magnetic mass susceptibility of $\mathrm{LiHoF}_{4}$ for the classical law with logarithmic correction $\chi_{c}^{m}=A t^{-1}\left|\log _{10}\right|^{1 / 3}$ and the power law $\chi_{c}^{m}=A^{\prime} t^{-\gamma} ; \chi_{c}^{m}$ is the mass susceptibility corrected for demagnetizing field effect, $\chi_{\max }$ is the maximum value of the measured mass susceptibility along the easy axis, $t$ is the reduced temperature $\left(T-T_{c}\right) / T_{c}$, and $\Delta=(1 / N) \sum_{i}\left|\chi_{\mathrm{th}}\left(t_{i}\right)-\chi_{\| 1}^{m}\left(t_{i}\right)\right|$ is the average difference between the theoretical susceptibility and the measured susceptibility on the $N$ experimental points in the temperature range $1.5273<T<1.544 \mathrm{~K}$.

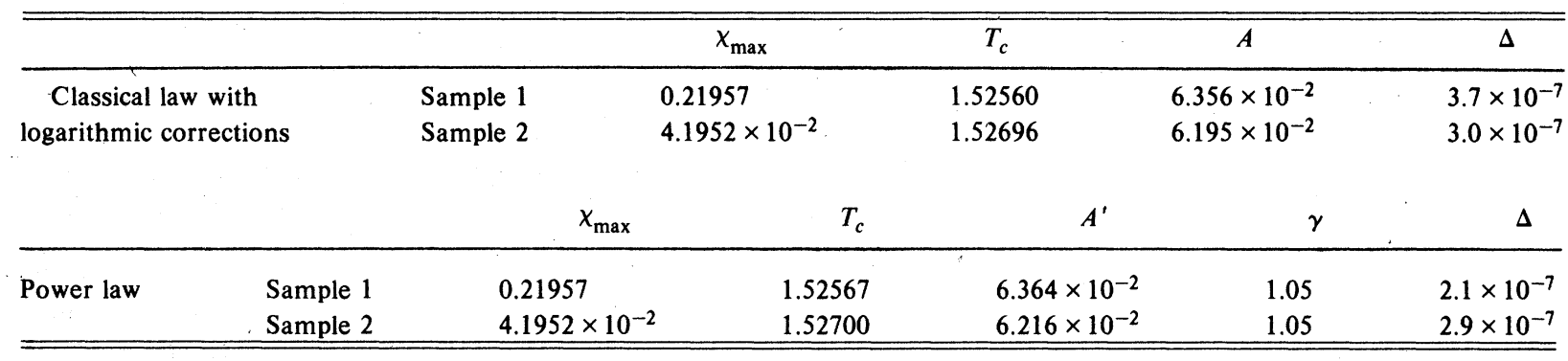

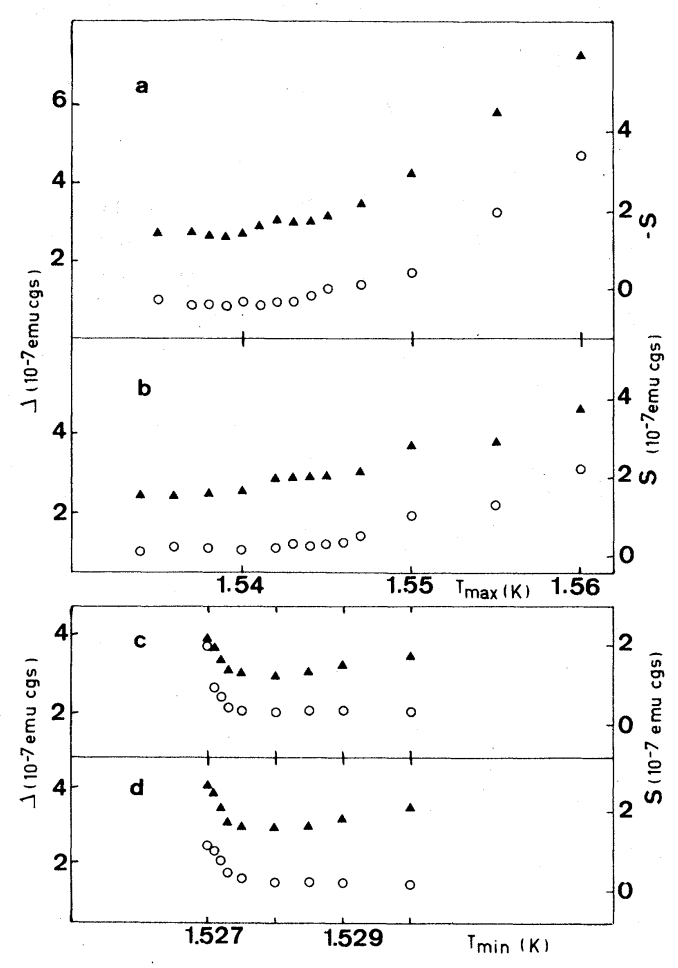

FIG. 6. $\Delta$ (black triangle) and $S$ (white circle) vs $T_{\max }$ and $T_{\text {min }} . \Delta$ is the average difference $(1 / N) \sum_{i}\left|\chi_{\mathrm{th}}\left(t_{i}\right)-\chi_{\|}^{m}\left(t_{i}\right)\right|$ and $S$ the algebraic sum $\sum_{i}\left[\chi_{\mathrm{th}}\left(t_{i}\right)-\chi_{\| 1}^{m}\left(t_{i}\right)\right]$ on the $N$ experimental points in the temperature range $T_{\min }<T<T_{\max }$. For a critical behavior with the values of critical parameters indicated in Table I for sample 2, we have plotted the following for a classical law with logarithmic corrections: (a) $\Delta$ and $-S$ vs $T_{\max }$ with $T_{\min }=1.5273 \mathrm{~K}$; (c) $\Delta$ and $S$ vs $T_{\min }$ with $T_{\max }=1.544 \mathrm{~K}$; for a power law: (b) $\Delta$ and $S$ vs $T_{\max }$ with $T_{\min }=1.5273 \mathrm{~K}$; (d) $\Delta$ and $S$ vs $T_{\min }$ with $T_{\max }=1.544 \mathrm{~K}$. rounding near $T_{c}$ and $t_{\max }$ does not exceed $10^{-2}$. The final values of the parameters which give the best fit to experimental data are reported in Table I for both samples 1 and 2 . The experimental data of the parallel susceptibility of sample 2 and the difference with the theoretical laws (7) and (8) versus temperature are given in Table II.

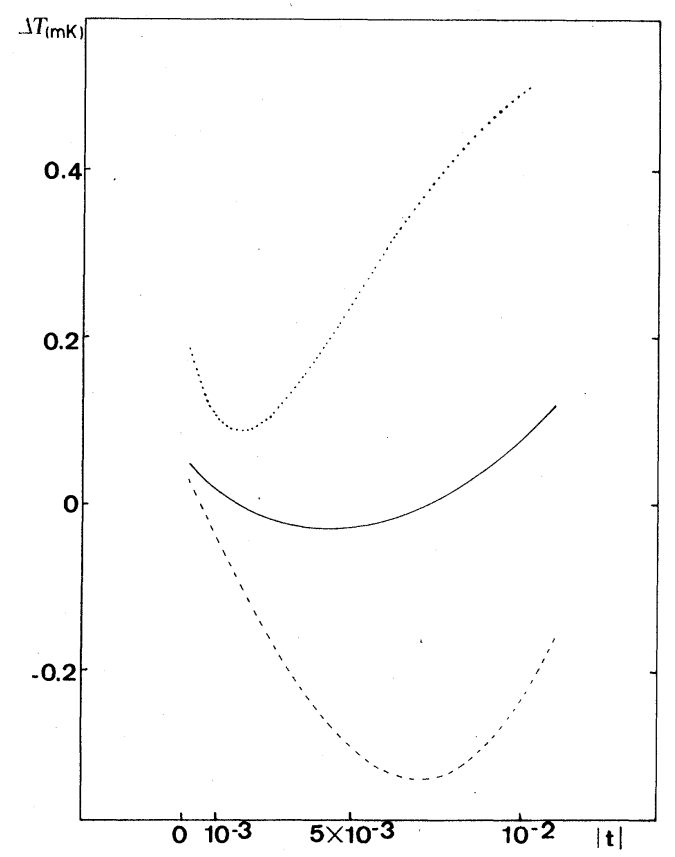

FIG. 7. $\Delta T=T_{c}\left(t^{\prime}-t\right)$ vs $t$ where $t$ is the reduced temperature $\left(T-T_{c}\right) / T_{c}$. We have plotted (full line) $\Delta T$ for our critical susceptibility measurements on $\mathrm{LiHoF}_{4}$ assuming $A t^{-1}\left|\log _{10} t\right|^{1 / 3}=A^{\prime} t^{\prime-\gamma}$. We have also plotted vs $t$ using the temperature difference between the power law and the classical law with logarithmic corrections for the specific-heat measurements of Ahlers et al. (Ref. 18) on $\mathrm{LiTbF}_{4}$ : dashed line for $T>T_{c}$ and dotted line for $T<T_{c}$. 
TABLE II. Experimental parallel susceptibility $\chi_{\|}$of sample 2 of $\mathrm{LiHoF}_{4}$ (spheroid sample of $4.836 \pm 0.002-\mathrm{mm}$ diam and $339.8 \pm 0.2-\mathrm{mg}$ weight) versus temperature. We have also reported the difference versus temperature between the experimental data and the two theoretical laws:

$\Delta X_{\ln }=A t^{-1}\left|\log _{10} t\right|^{1 / 3}-X_{\|} ; \Delta X_{\text {pow }}=A^{\prime} t^{-\gamma}-X_{\|}$; with the values of the parameters indicated in Table I.

\begin{tabular}{|c|c|c|c|}
\hline$T$ & $x\left(10^{-2}\right.$ emu cgs $)$ & $\Delta \chi_{\ln }\left(10^{-6}\right.$ emu cgs $)$ & $\Delta \chi_{\text {pow }}\left(10^{-6}\right.$ emu cgs $)$ \\
\hline 1.5434 & 1.41739 & -11.1 & -5.8 \\
\hline 1.5415 & 1.41841 & -12.6 & -9.6 \\
\hline 1.5396 & 1.41938 & -8.6 & -7.7 \\
\hline 1.5377 & 1.42030 & -1.5 & -1.9 \\
\hline 1.5359 & 1.42120 & 1.3 & 0 \\
\hline 1.53413 & 1.42289 & 9.8 & 8.3 \\
\hline 1.52895 & 1.42464 & -0.4 & -0.2 \\
\hline 1.52707 & 1.42535 & 13.0 & 14.0 \\
\hline 1.52529 & 1.42545 & 7.0 & 7.0 \\
\hline 1.52438 & 1.42550 & 2.0 & 2.0 \\
\hline 1.52356 & 1.42552 & 0.0 & 0.0 \\
\hline 1.5218 & 1.42552 & 0.0 & 0.0 \\
\hline 1.54325 & 1.41741 & -5.1 & 0 \\
\hline 1.5421 & 1.41801 & -3.9 & -0.2 \\
\hline 1.5410 & 1.41854 & 1.1 & 3.4 \\
\hline 1.53983 & 1.41918 & -1.3 & -0.2 \\
\hline 1.53868 & 1.41978 & -1.3 & -0.9 \\
\hline 1.53726 & 1.42051 & -0.2 & -0.9 \\
\hline 1.53640 & 1.42096 & -0.8 & -1.9 \\
\hline 1.53528 & 1.42154 & -1.3 & -2.8 \\
\hline 1.53433 & 1.42199 & 1.7 & 0.4 \\
\hline 1.53348 & 1.42242 & 1.7 & 0.2 \\
\hline 1.53251 & 1.42295 & -3.2 & -4.7 \\
\hline 1.53176 & 1.42331 & -2.6 & -4.1 \\
\hline 1.53101 & 1.42370 & -4.5 & -5.6 \\
\hline 1.53013 & 1.42404 & 3.6 & 3.0 \\
\hline 1.52959 & 1.42428 & 5.8 & 5.6 \\
\hline 1.52897 & 1.42460 & 2.8 & 3.0 \\
\hline 1.52867 & 1.42477 & -0.4 & 0.1 \\
\hline 1.52825 & 1.42500 & -4.7 & -3.9 \\
\hline 1.52788 & 1.42513 & -3.0 & -1.7 \\
\hline 1.52762 & 1.42520 & 4.3 & 5.6 \\
\hline 1.52722 & 1.42535 & 6.0 & 8.0 \\
\hline 1.52687 & 1.42539 & 13.0 & 13.0 \\
\hline 1.52600 & 1.42545 & 7.0 & 7.0 \\
\hline 1.52540 & 1.42545 & 7.0 & 7.0 \\
\hline 1.52360 & 1.42550 & 2.0 & 2.0 \\
\hline 1.5421 & 1.41796 & 4.7 & 4.1 \\
\hline 1.5420 & 1.41888 & 9.0 & 10.5 \\
\hline 1.5385 & 1.41983 & 3.9 & 4.1 \\
\hline 1.53685 & 1.42064 & 8.1 & 7.3 \\
\hline 1.5350 & 1.42164 & 2.1 & 0.9 \\
\hline 1.5332 & 1.42890 & 9.4 & 7.9 \\
\hline 1.5316 & 1.42338 & -1.3 & -2.4 \\
\hline 1.5299 & 1.42419 & -0.4 & -2.6 \\
\hline 1.5290 & 1.42462 & -0.6 & -0.2 \\
\hline 1.5281 & 1.42505 & -2.1 & -1.1 \\
\hline 1.52765 & 1.42520 & 3.0 & 4.3 \\
\hline
\end{tabular}


TABLE II. (Continued)

\begin{tabular}{|c|c|c|c|}
\hline$T$ & $x\left(10^{-2}\right.$ emucgs $)$ & $\Delta x_{\ln }\left(10^{-6}\right.$ emu cgs $)$ & $\Delta x_{\text {pow }}\left(10^{-6}\right.$ emu cgs $)$ \\
\hline 1.52737 & 1.42526 & 8.8 & 10.3 \\
\hline 1.52782 & 1.42518 & -2.4 & -1.3 \\
\hline 1.52721 & 1.42530 & 12.0 & 13.0 \\
\hline 1.52688 & 1.42541 & 11.0 & 11.0 \\
\hline 1.52601 & 1.42548 & 4.0 & 4.0 \\
\hline 1.52501 & 1.42550 & 2.0 & 2.0 \\
\hline 1.52482 & 1.42550 & 2.0 & 2.0 \\
\hline 1.52303 & 1.42552 & 0.0 & 0.0 \\
\hline 1.51967 & 1.42554 & -2.0 & -2.0 \\
\hline 1.5428 & 1.41758 & $1: 7$ & 6.2 \\
\hline 1.53304 & 1.42261 & 4.5 & 3.0 \\
\hline 1.53247 & 1.42293 & 8.3 & -0.6 \\
\hline 1.53127 & 1.42349 & 4.1 & 3.0 \\
\hline 1.53063 & 1.42383 & 1.1 & 0.2 \\
\hline 1.53014 & 1.42406 & 1.1 & 0.4 \\
\hline 1.52939 & 1.42443 & 0.2 & 0.2 \\
\hline 1.52903 & 1.42460 & 0. & 0.2 \\
\hline 1.52861 & 1.42481 & -1.9 & -1.3 \\
\hline 1.52822 & 1.42496 & 0.9 & 1.7 \\
\hline 1.52790 & 1.42513 & -1.7 & -0.6 \\
\hline 1.52768 & 1.42518 & 3.9 & 5.1 \\
\hline 1.52746 & 1.42528 & 3.0 & 5.0 \\
\hline 1.52726 & 1.42533 & 7.0 & 8.0 \\
\hline 1.52710 & 1.42535 & 11.0 & 13.0 \\
\hline 1.52695 & 1.42537 & 15.0 & 15.0 \\
\hline 1.52671 & 1.42539 & 13.0 & 13.0 \\
\hline$' 1.52607$ & 1.42541 & 11.0 & 11.0 \\
\hline 1.52426 & 1.42548 & 4.0 & 4.0 \\
\hline 1.52151 & 1.42552 & 0.0 & 0.0 \\
\hline
\end{tabular}

With the parameters of Table I for sample 2 we obtain in the temperature range $1.5273<T<1.544$

$$
\begin{aligned}
s^{2} & =\frac{1}{N-F} \sum_{i}\left[\chi_{\mathrm{th}}\left(t_{i}\right)-\chi_{\| 1}\left(t_{i}\right)\right]^{2} \\
& =2.15 \times 10^{-13} \text { emu cgs },
\end{aligned}
$$

where $F$ is the number of fit parameters and $\chi^{2}=1.34$.

A similar fit procedure has been adopted with the power law. ${ }^{8}$ In this case there is an additional parameter $\gamma$, and $\Delta\left(A^{\prime}\right)$ curves were systematically drawn for different values of $T_{c}$ and $\gamma$. The resulting best values of these parameters are given in Table $I$ and we obtain for sample 2: $s^{2}=1.91 \times 10^{-13}$ emu cgs and $\chi^{2}=1.19$.

A slight change of $\chi_{\max }$ does not appreciably affect the amplitudes $A$ and $A^{\prime}$ and the exponent $\gamma$ but shifts $T_{c}$ of the same quantity for both laws (7) and (8). The observed small discrepancies between the samples 1 and 2 are probably due to their completely different origin and especially to different impurity contents.
We have also studied the influence of $T_{\min }$ and $T_{\max }$ on the average difference

$$
\Delta=\frac{1}{N} \sum_{i}\left|\chi_{\mathrm{th}}\left(t_{i}\right)-\chi_{\|}^{m}\left(T_{i}\right)\right|
$$

and on the algebraic sum

$$
S=\sum_{i}\left[\chi_{\mathrm{th}}\left(t_{i}\right)-\chi_{\|}^{m}\left(t_{i}\right)\right]
$$

all other parameters being fixed at their values in Table I. Figure 6(a) shows $\Delta$ and $-S$ vs $T_{\max }$. They remain small until $T_{\max } \simeq 1.544$ and strongly increase when temperature exceeds this value. The same phenomenon is observed for both laws (7) and (8). This $T_{\max }=1.544 \mathrm{~K}$ which corresponds to a relative temperature $t_{\max }=1.1 \times 10^{-2}$ can be considered as the upper limit of the critical region. This extension of the critical region is consistent with the values reported for $\mathrm{LiTbF}_{4}\left(t_{\max } \sim 10^{-2}\right),{ }^{18}$ and $\mathrm{GdCl}_{3}$ $\left(t_{\max }=1.15 \times 10^{-2}\right) .{ }^{26}$ In Fig. $6(\mathrm{c})$, we have plotted $\Delta$ and $S$ vs $T_{\min }$ for a fixed $T_{\max }$ value of $1.544 \mathrm{~K}$. In 
sample 2 , the rounding effect starts at $T_{\min }=1.5273$ $\mathrm{K}$ which corresponds to $t_{\min }=2 \times 10^{-4}$. It appears that the rounding effect is smaller in this $\mathrm{LiHoF}_{4}$ samples than that observed on $\mathrm{LiTbF}_{4}\left(t_{\min }=10^{-3}\right)$ and $\mathrm{GdCl}_{3}\left(t_{\min }=1.5 \times 10^{-3}\right)$.

Finally, we are not able to discriminate between the laws (7) and (8) on the basis of the present experimental data. The average difference of the best fit to the data is, respectively, equal to $3.0 \times 10^{-7}$ and $2.9 \times 10^{-7}$ emu cgs for the laws (7) and (8) on sample 2. Assuming $A t^{-1}\left|\log _{10} t\right|^{1 / 3}=A^{\prime} t^{\prime-\gamma}$, we have calculated the temperature difference $\Delta T=T_{c}\left(t^{\prime}-t\right)$ vs $T$ (Fig. 7). The maximum $\Delta T$ value is equal to $0.1 \mathrm{mK}$ in the critical temperature range. Since the $T_{58}$ temperature scale has absolute errors of about $2 \mathrm{mK}$ and an internal point to point roughness of $0.1 \mathrm{mK},{ }^{27}$ it would be rather difficult to improve the thermometry in the actual temperature range and to bring forward discrimination between the considered critical laws.

\section{CONCLUSION}

The present susceptibility measurements confirm that $\mathrm{LiHoF}_{4}$ is a fairly good uniaxial dipolar ferromagnet. The parallel susceptibility is accurately fitted by the classical law with logarithmic corrections predicted by Larkin and Khmel'nitskii. Nevertheless we could not discriminate between this theoretical law and a power law with a critical exponent rather close to 1 . This discrimination would require an improved temperature scale with a point to point roughness better than $0.01 \mathrm{mK}$ at $1.5-1.6 \mathrm{~K}$. Magnetization and specific-heat measurements in the critical region would be useful to complete this study. Further work is in progress.
${ }^{1}$ R. E. Thoma, G. D. Brunton, R. A. Penneman, and T. K. Keenan, Inorg. Chem. 9, 1096 (1970).

${ }^{2}$ E. P. Chicklis and C. S. Naiman, IEEE J. Quantum Electron. QE 8, 535 (1972).

${ }^{3}$ R. K. Watts and W. C. Holton, Solid State Commun. 9, 137 (1971).

${ }^{4}$ H. P. Jenssen, A. Linz, R. P. Leavitt, C. A. Morrison, and D. E. Westman, Phys. Rev. B 11, 92 (1975); J. E. Miller and E. J. Sharp, J. Appl. Phys. 41, 4718 (1970); A. L. Harmer, A.Linz, and D. R. Gabbe, J. Phys. Chem. Solids 30, 1483 (1969); M. R. Brown, K. G. Rooth, and W. A. Shand, J. Phys. C 2, 593 (1969).

${ }^{5}$ J. P. Sattle and J. Nemarich, Phys. Rev. B 4, 1 (1971); J. Margariño, J. Tuchendler, J. P. D'Haenens, and A. Linz, Phys. Rev. B $\underline{13}, 2805$ (1976).

6J. Margariño and J. Tuchendler, Physica 86-88B, 1233 (1977).

${ }^{7}$ I. Laursen and L. M. Holmes, J. Phys. C 7, 3765 (1974).

${ }^{8}$ S. K. Misra and J. Felsteiner, Phys. Rev. B 15,4309 (1977).

${ }^{9}$ L. M. Holmes, T. Johansson, and H. J. Guggenheim, Solid State Commun. 12, 993 (1973).

${ }^{10}$ P. E. Hansen, T. Johansson, and R. Nevald, Phys. Rev. B 12,5315 (1975).

${ }^{11}$ A. H. Cooke, D. A. Jones, J. F. A. Silva, and M. R. Wells, J. Phys. C 8,4083 (1975).
12P. Beauvillain, J.-P. Renard, and P. E. Hansen, J. Phys. C 10, L709 (1977).

${ }^{13} \mathrm{See}$, for instance, K. G. Wilson and J. Kogut, Phys. Rev. C 12,75 (1974).

${ }^{14}$ I. A. Larkin and D. E. Khmel'nitskii, Zh. Eksp. Teor. Fiz. 65, 2087 (1969) [Sov. Phys.-JETP 29, 1123 (1969)].

${ }_{15}$ A. Aharony, Phys. Rev. B 8, 3363 (1973).

${ }^{16} \mathrm{E}$. Brézin and J. Zinn-Justin, Phys. Rev. B 13, 251 (1976).

${ }^{17}$ A. Aharony and B. I. Halperin, Phys. Rev. Lett. 35, 1308 (1975).

${ }^{18}$ G. Ahlers, A. Kornblit, and H. J. Guggenheim, Phys. Rev. Lett. 34, 1227 (1975).

${ }^{19}$ J. Als-Nielsen, Phys. Rev. Lett. 37, 1161 (1976).

20J. A. Griffin, J. D. Litster, and A. Linz, Phys. Rev. Lett. 38, 251 (1977).

${ }^{21}$ B. Lécuyer, Thèse CNAM (Paris, 1969) (unpublished).

${ }^{22}$ ATNE, Z. A. Courtaboeuf, 91400 Orsay, France.

${ }^{23} \mathrm{Ge}$ resistor model N2, Scientific Instruments, Lake Worth, Texas.

${ }^{24}$ Pressure gauge, Texas Instruments, Dallas Road, Bedford, Texas.

25J. Magariño and J. Tuchendler (unpublished).

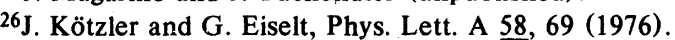

${ }^{27}$ J. R. Clement, Temperature, (Reinhold, New York, 1962), Vol. 3, p. 67. 